This article was published in Physical Chemistry Chemical Physics, 17(29), 18971-18979, 2015

http://dx.doi.org/10.1039/c5cp02717a

\title{
Structural Characterization of Functionalized Gold Nanoparticles for Drug Delivery in Cancer Therapy: a NMR Based Approach
}

Sílvia C. Coelho ${ }^{a}$, Maria Rangel ${ }^{b}$, Maria C. Pereira ${ }^{a}$, Manuel A.N. Coelho ${ }^{a *}$, Galya Ivanova ${ }^{c *}$

\begin{abstract}
In the present paper, we report results from a study of the structure and physicochemical properties of gold nanoparticles modified with poly(ethylene glycol) (PEG) designed for drug delivery of the proteasome inhibitor Bortezomib (BTZ) in cancer therapy. A number of advanced analytical techniques were used to define important physicochemical characteristics such as composition, structure, surface properties, particle size and morphology. A new approach based on detailed NMR studies was employed to define specific intermolecular interactions and mechanisms of drug immobilization and location into surface modified gold nanoparticles (AuNPs). Particularly important information was gained from analysis of NMR spectroscopic parameters such as spectral line shape, translation diffusion, nuclear Overhauser effect (NOE) and spin-lattice relaxation (T1). The results confirmed the coexistence of two different types of BTZ inclusion into polyethylene glycol coated gold nanoparticles: (i) association with the polymer chains by weak H-bonds and/or dipole-charge interactions and (ii) adsorption on the surface of the gold nanoparticles. The results allowed for determination of the overall structure of Bortezomib loaded PEG coated AuNPs, which is related to the therapeutic drug efficacy and activity in the treatment of cancer.
\end{abstract}

\section{Introduction}

Nanoscience and nanotechnology hold great promise for biomedical applications and have the potential to generate entirely novel and highly effective therapeutic agents based on nano-systems such as nanoparticles (NPs). One of the main applications of nanotechnology to medicine is the development of NPs as drug-delivery systems (DDS) for cancer therapy. The main challenge in designing such systems is to overcome several limitations of conventional chemotherapeutics and improve their therapeutic index through decrease in toxic side effects and levels of multidrug resistance, and increase in drug efficacy. ${ }^{1,2}$ Effective drug-delivery (DD) requires successful navigation of carriers through complex biological processes in the body. The therapeutic efficacy of DDS is therefore affected by the physicochemical properties of nano-formulations and the impact of these on the transport, binding and metabolism of the drug in the body. ${ }^{1-3}$ The size, shape and surface chemistry of DDS are properties that affect their biodistribution, circulation time, renal clearance and endocytosis. ${ }^{4-6}$ On the other hand, the composition and structure of DDS affect the therapeutic efficacy characteristics such as capacity for sustained drug release and transport through bio-membranes, targeted drug delivery and bioavailability. ${ }^{3}$

Over the last decade, it has been shown that colloidal particles are promising DDS, which can potentially overcome physiological barriers and target the drugs to specific cells/tissues or intracellular compartments, as well as internalization in different cell cycle phases. ${ }^{1,2,7}$ DDS used in therapy may be composed of liposomes, polymeric nanoparticles, dendrimers, metal nanoparticles and molecular targeted nanoparticles. ${ }^{2,3}$ Among the broad diversity of nanocarriers, gold nanoparticles (AuNPs), due to their unique chemical, physical, pharmacological and optical properties have demonstrated great potential for early detection, diagnosis and effective treatment of cancer. ${ }^{3,5}$ The remarkable properties of AuNPs, such as size- and shape-dependent optical and electronic features, high surface-to-volume ratio, excellent biocompatibility and chemical stability allow them to be versatile drug delivery platforms for cancer therapy and diagnosis. ${ }^{8-11}$ AuNPs, in a variety of shapes with sizes ranging from $1 \mathrm{~nm}$ to more than $100 \mathrm{~nm}$ can be easily synthesized and modified. ${ }^{12}$ AuNPs, being biocompatible and easily conjugated to drugs and biomolecules are used in a wide variety of biomedical applications such as chemotherapy, photothermal, photodynamic and radiation therapy (Figure 1). ${ }^{10-12}$ 


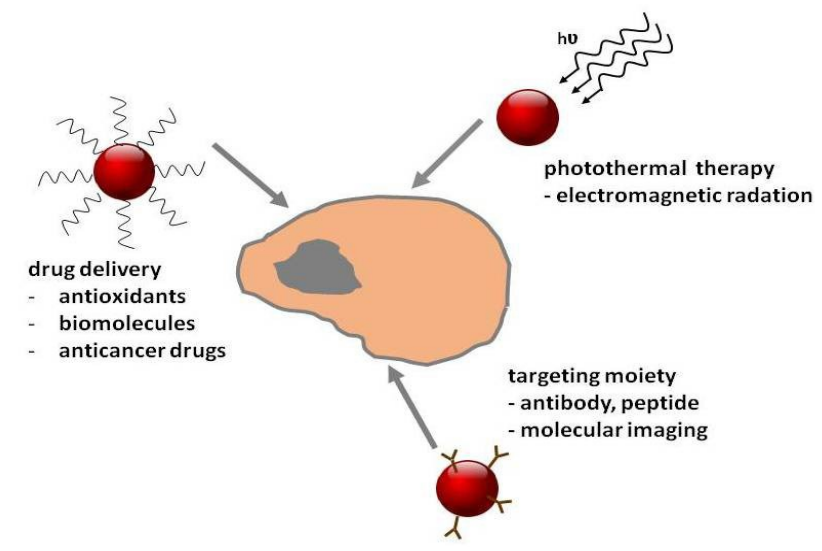

Figure 1. Functionalized gold nanoparticles for cancer therapies and diagnosis.

Several reports reveal the potential of AuNPs as labels in cancer diagnostics and detection, and their ability to significantly improve the treatment efficacy in photothermal cancer therapy. ${ }^{10,13-15}$ Kuo et al. have recently shown that the conjugation of AuNPs with fluorescent dye/photosensitizers results in a promising diagnostic probe and when combined with photodynamic and photothermal therapies provoke killing of cancer cells. ${ }^{16}$ Results from studies of AuNPs functionalized with anti-cancer drugs demonstrate the capacity of these systems for targeted drug delivery, overcoming multidrug resistance and finally cancer cell apoptosis. ${ }^{17-19}$

Recently, it has become apparent that the surface functionality and morphology of AuNPs can affect the overall NP behaviour and important therapeutic characteristics such as the cellular uptake, intracellular release and distribution in sub-cellular compartments, and circulation life time. ${ }^{20-23}$ The modification of the surface properties of AuNPs has the potential to control the permeation, distribution and retention of drugs in tumors, and hence their therapeutic efficacy. ${ }^{24-28}$ Surface modifications of AuNPs with poly(ethylene glycol) (PEG) could improve their stability and in vivo performance and it was shown that the PEGylation of AuNPs increase the circulation life time and avoid their detection and clearance by the reticuloendothelial system. ${ }^{29-31}$ Thus, the structure and physicochemical characteristics of AuNPs appear to be key factors that could affect their in vivo behaviour and effectiveness as DDS. Moreover, the type and strength of the intermolecular interactions in DDS is of fundamental importance and govern their capacity for efficient, controlled and targeted drug delivery. ${ }^{1-3}$ To exploit the potential of AuNPs in drug delivery, a comprehensive study of their physicochemical characteristics is necessary, for rational design and development of unique platforms for efficient drug delivery for cancer therapy.

Nowadays, a number of important spectroscopic and imaging techniques can be used for physicochemical characterization of nano-formulations for DD. ${ }^{32}$, ${ }^{33}$ Among them, Nuclear Magnetic Resonance (NMR) spectroscopy holds a prominent place for being non-destructive, highly reproducible and sensitive to structural details of molecules and molecular conjugates, which can be used for both qualitative and quantitative characterization purposes. A variety of well-established NMR experiments can provide valuable information on the molecular size, shape, dynamics, chemical structure, intermolecular interactions, binding and exchange processes in complex nano-systems. ${ }^{33-35}$ NMR allows for the in situ nondestructive analysis of nano-systems and can be particularly useful in distinguishing between free and interacting with and/or bound to NP species, and to quantify the distribution of these species. In this context, diffusion ordered spectroscopy (DOSY) and nuclear Overhauser effect (NOE) spectroscopy, being particularly sensitive to structural and dynamical changes, have become invaluable tools for studies of the molecular transport, interactions and binding processes in complex systems. ${ }^{36-38}$ Recently published results have proved the capability of NMR spectroscopy as a method for structural analysis of nano-therapeutics. ${ }^{34}$, 39,40

We have recently designed gold nanoparticles surface modified with functionalized poly(ethylene glycol) (PEGAuNPs) for targeted delivery of the highly toxic anticancer drug Bortezomib (BTZ) (Figure 2). ${ }^{41,42}$ 


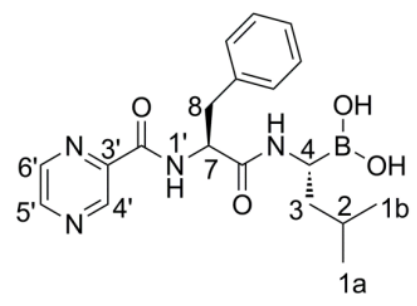

Figure 2. Chemical structure of Bortezomib (BTZ).

BTZ is a boronic dipeptide that inhibits the function of the 26S proteasome and is approved by the U.S. Food and Drug Administration (FDA). ${ }^{43-45}$ The uptake mechanism of the newly designed BTZ loaded AuNPs in pancreatic and prostate cell lines was assessed by laser scanning confocal microscopy (LSCM). ${ }^{41,42}$ The results have demonstrated the ability of cancer cells to internalize AuNPs together with BTZ and it was confirmed that these nanoparticles are effective delivery systems, increasing the drug internalization and retention in cancer cells. ${ }^{41,42}$

In the present paper, we report results based on NMR and FTIR (Fourier transform infrared spectroscopy) structural analysis and physicochemical characterization of surface modified gold nanoparticles as a vehicle for delivery of Bortezomib in cancer therapy. Physicochemical characteristics such as composition, particle size and morphology, surface properties, intermolecular interactions and mechanisms of drug immobilization and location in PEGAUNPs have been assessed. The results allow for determination of the overall structure of drug-loaded PEGAuNPs, which is related to the drug efficacy and reactivity.

\section{Experimental Section}

\section{Reagents and chemicals}

Bortezomib was purchased from Selleck Chemicals LLC (Germany). Trisodium citrate dehydrate and tetrachloroauric (III) acid ( $\mathrm{HAuCl} 4$; 99.99\% trace metals basis, 30 wt\% in dilute $\mathrm{HCl}$ ), Phosphate-buffered saline (PBS) were purchased from Sigma-Aldrich (Germany). 2-(2-[2-(11-mercapto-undecyloxy)-ethoxy)ethoxy]-ethoxy)-ethoxy-acetic acid (HS-C11EG3-OCH2COO) (molecular weight 394.57 Da) was purchased from Prochimia (Poland).

\section{Synthetic procedures}

\section{Synthesis of functionalized gold nanoparticles}

AuNPs were synthesized by reduction of $\mathrm{HAuCl}_{4}$ using sodium citrate, the Turkevich-Frens method. ${ }^{44}$ AuNPs were further coated with 2-(2-[2-(11-mercapto-undecyloxy)-ethoxy)-ethoxy]-ethoxy)-ethoxy-acetic acid (PEG), at a molar ratio of 1:1000. The preparation occurred over 1 hour, by constant stirring and at room temperature. The obtained PEGylated AuNPs (PEGAuNPs) were centrifuged at 13400 rpm for 10 minutes to remove the unbounded PEG. This step was performed three times.

Preparation of Bortezomib loaded PEGylated gold nanoparticles (PEGAuNPs+BTZ)

A stock solution of BTZ in dimethyl sulfoxide was prepared at room temperature. Aliquots of this solution were added to the PEGAuNPs colloidal suspension to give a final BTZ concentration of $50 \%$ of the PEG amount. To perform the NMR analyses, the intact colloidal suspensions of both unloaded and drug-loaded PEGAUNPs were additionally concentrated by centrifugation at $14500 \mathrm{rpm}$ for $10 \mathrm{~min}$.

\section{Characterization techniques}

Dynamic light scattering (DLS)

Size distribution and zeta potential of the nanoparticle suspensions were determined in deionised water, at 25 o $C$ by dynamic light scattering and laser doppler velocimetry, respectively, using a Zetasizer Nano ZS (Malvern Instruments Ltd., Malvern, UK).

\section{Transmission electron microscopy (TEM)}

Five $\mu \mathrm{L}$ of the PEGAuNPs suspension was placed on a carbon formvar-coated grid and left to adsorb for 2 min. After adsorption, the grid was washed with deionized (DI) water to remove the excess suspension. The grid was visualized by transmission electron microscopy (TEM) using a Zeiss microscope (Jeol JEM 1400 with Gatan Orius camara) operated at $60 \mathrm{kV}$. 


\section{UV-Vis spectroscopy}

UV-Vis spectroscopy measurements of PEGAuNPs and PEGAuNPs+BTZ were carried out on a Shimadzu UV1700 PharmaSpec spectrophotometer with a $1 \mathrm{~cm}$ quartz cuvette.

\section{FTIR spectroscopy}

FTIR transmission spectra were recorded with an ALPHA ATR FTIR Spectrometer (Bruker) in the spectral range $4000-400 \mathrm{~cm}^{-1}, 64$ scans were recorded with a resolution of $4 \mathrm{~cm}^{-1}$, at room temperature.

\section{NMR spectroscopy}

The NMR spectra were recorded on a Bruker Avance III 400 and/or Bruker Avance III 600 HD spectrometer, operating at 400.15 and $600.13 \mathrm{MHz}$ for protons, respectively. The NMR spectrometers equipped with pulse gradient units, are capable of producing magnetic field pulsed gradients in the $z$-direction of 53 and $50 \mathrm{G} / \mathrm{cm}$, respectively. The NMR measurements were carried out using standard BRUKER pulse sequences, in water solution with $10 \%$ deuterium oxide $\left(\mathrm{H}_{2} \mathrm{O}: \mathrm{D}_{2} \mathrm{O}\right)$ at a temperature of $300 \mathrm{~K}$. Sodium trimethylsilyl$[2,2,3,3 \mathrm{~d} 4]$-propionate (TSP) was used as an internal standard for both the chemical shift and the diffusion measurements. ${ }^{1} \mathrm{H}$ NMR experiments were performed with water suppression using excitation sculpting with gradients ${ }^{45}$, an acquisition time of $1.36 \mathrm{~s}$, a relaxation delay of $2 \mathrm{~s} .128$ or 256 transients of a spectral width of $10000 \mathrm{~Hz}$ were collected into $32 \mathrm{k}$ time domain points. Two dimensional ${ }^{1} \mathrm{H} /{ }^{1} \mathrm{H}$ correlated spectra (COSY) were recorded in phase sensitive mode and with water suppression, a relaxation delay of $2 \mathrm{~s}, 32$ or 64 scans, giving a total of $2 \mathrm{k}$ data points in $\mathrm{F} 2$ and 256 data points in F1, over a spectral width of $10000 \mathrm{~Hz}$. $2 \mathrm{D}{ }^{1} \mathrm{H} /{ }^{13} \mathrm{C} \mathrm{HSQC}$ and $\mathrm{HMBC}$ experiments were carried out, with a spectral width of ca. $10000 \mathrm{~Hz}$ for ${ }^{1} \mathrm{H}$ and $32000 \mathrm{~Hz}$ for ${ }^{13} \mathrm{C}$, with a relaxation delay of $1.5 \mathrm{~s}$ and a Fourier transform (FT) size of $2 \mathrm{k} \times 1 \mathrm{k}$.

Saturation Transfer Difference (STD) ${ }^{1} \mathrm{H}$ NMR spectra were recorded at $300 \mathrm{~K}$ on a Bruker Avance III 400 spectrometer, using a pseudo 2D pulse sequence for saturation transfer difference, with a shaped pulse train for saturation, alternating between on and off resonance and with water suppression using excitation sculpting with gradients. ${ }^{46,47}$ The PEG coating layer of AuNPs was saturated on-resonance at $3.65 \mathrm{ppm}$ $\left(\mathrm{OCH}_{2}\right)$ and off-resonance at $30 \mathrm{ppm}$, with a cascade of 50 selective Gaussian shaped pulses of $50 \mathrm{~ms}$ duration, with a delay between the pulses of $1 \mathrm{~ms}$, total duration of the saturation time of $2.5 \mathrm{~s}$ and a relaxation time of $3.5 \mathrm{~s}$.

The spin-lattice relaxation rates were measured using the inversion-recovery pulse sequence, $\left(180^{\circ}-\tau\right.$ $\left.90^{\circ}\right)$. Twenty $\tau$ increments were used for the experiments, with values between 0.01 and $10.0 \mathrm{~s}$. In all cases the relaxation delay was $10 \mathrm{~s}$. Relaxation times were calculated by exponential regression analysis of recovery curves of longitudinal magnetization components.

Proton registered diffusion-ordered NMR $\left({ }^{1} \mathrm{H}\right.$ DOSY) experiments were acquired using the bipolar longitudinal eddy current delay (BPPLED - Bipolar Pulsed Field Gradient Longitudinal Eddy Delay) pulse sequence $^{48}$ with pre-saturation of the water resonance during the relaxation delay. The experimental conditions (amount of the solute and solvent, a temperature of $300 \mathrm{~K}$, no sample rotation and air flow of $535 \mathrm{~L} / \mathrm{h}$ to avoid convections in the samples) for all DOSY experiments were kept constant. Before the NMR experiments, the temperature was equilibrated and maintained at $300 \mathrm{~K}$, as measured using the spectrometer thermocouple system. Typically, in each experiment 16 spectra of 16k data points and 128 or 256 scans were collected, with values for the duration of the magnetic field pulse gradients $(\delta)$ of 2.4 or $3.2 \mathrm{~ms}$, diffusion times $(\Delta)$ of $100-200 \mathrm{~ms}$ and an eddy current delay set to $5 \mathrm{~ms}$. The pulse gradient $(\mathrm{g})$ was incremented from 2 to $98 \%$ of the maximum gradient strength in a linear ramp. The spectra were processed with the Bruker Topspin software package (version 3.2) and the diffusion coefficients calculated by exponential fitting of the data belonging to individual columns of the 2D matrix. Between 3 and 5 different measurements were performed for thedetermination of each diffusion coefficient. The diffusion coefficients (D) were all scaled to the absolute D value obtained for TSP $\left(6.68 \times 10^{-10} \mathrm{~m}^{2} \mathrm{~s}^{-1}\right.$, calculated standard deviation of $3.1 \times 10^{-3}$ ) used as an internal reference.

\section{Results and Discussion}

AuNPs with a mean hydrodynamic diameter of $35 \mathrm{~nm}$ were prepared as an aqueous dispersion by the Turkevitch method and further functionalized with a thiolated PEG layer functionalized with carboxylate groups to form PEGAuNPs (Table 1). The PEGAuNPs stability in presence of PBS was evaluated by hydrodynamic diameter and zeta potential measurements (Table 1). In presence of PBS, it was not observed significant changes in zeta potential and hydrodynamic diameter of PEGAuNPs. This effect was expected by the fact that PEG confers steric stabilization to the AuNPs. Scheme 1 represents the method of preparation of BTZ loaded PEG coated gold nanoparticles (PEGAuNPs+BTZ). 


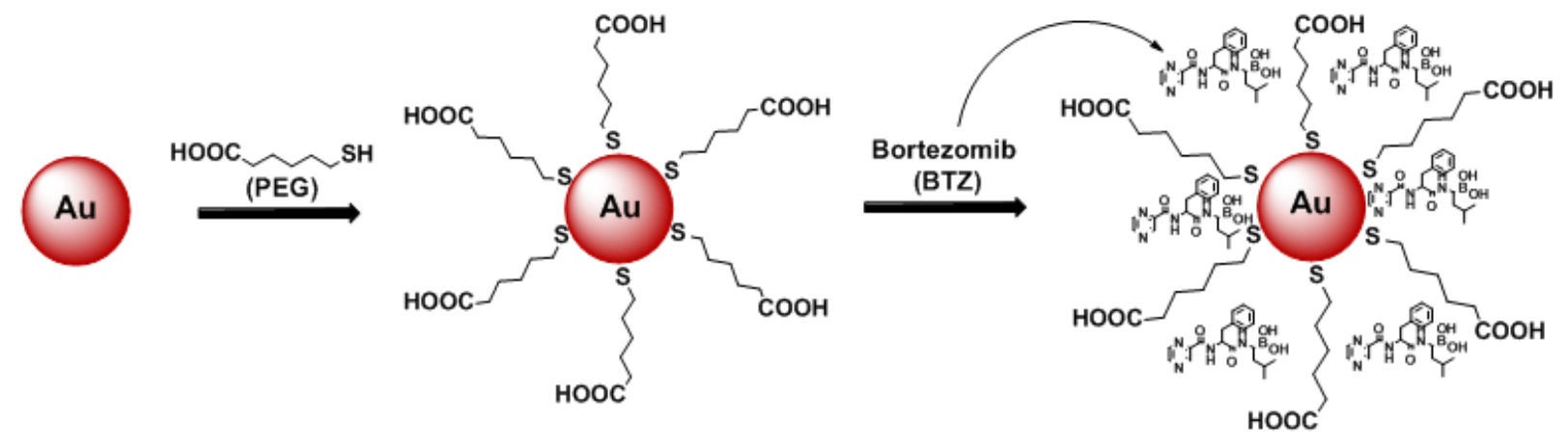

Scheme 1. Preparation of BTZ loaded PEGAuNPs.

Figure 3 shows a typical TEM image of PEGAuNPs. The particles in AuNPs and PEGAuNPs were found to adopt mostly spherical shapes with an average size around $40 \mathrm{~nm}$. The TEM images confirmed the small size of PEGAuNPs and low level of gold NPs aggregation. The size of the nanoparticles was additionally determined by DLS (Table 1) and the data corroborated the results obtained by TEM. The analysis of the DLS data showed that the BTZ loading of PEGAuNPs results in particles (PEGAuNPs+BTZ) with a size similar to those of unloaded (PEGAuNPs) particles (Table 1).

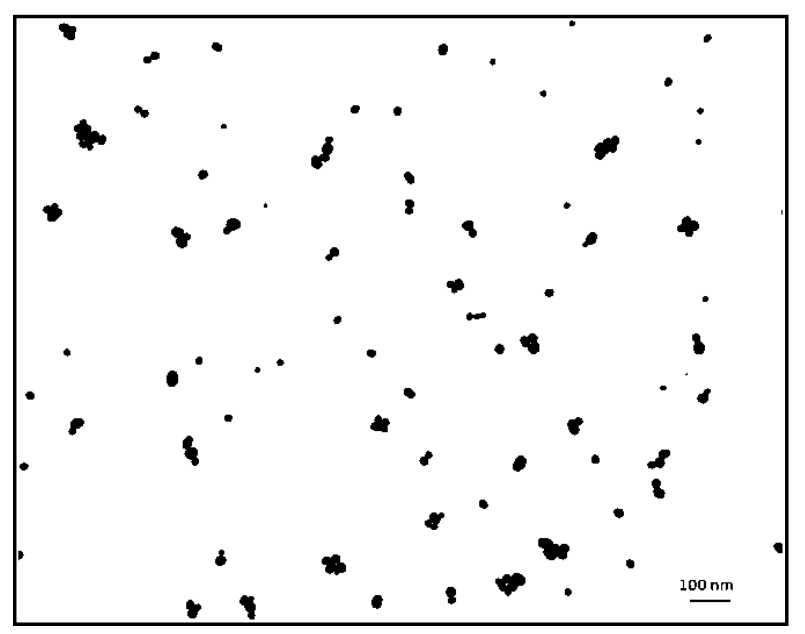

Figure 3. TEM image of PEGAuNPs. Scale bar is $100 \mathrm{~nm}$.

The formation of negatively charged gold nanoparticles was confirmed by the zeta-potential value of -40 $\mathrm{mV}$. The surface modification of these nanoparticles by PEGylation did not significantly change the zetapotential of the AuNPs $(-41 \mathrm{mV})$, thus preventing aggregation during long-term storage. After BTZ addition to PEGAuNPs, a significant change in zeta-potential was observed (from $-41 \mathrm{mV}$ to $-18 \mathrm{mV}$ ) revealing the binding and/or adsorption of drug molecules on the gold nanoparticles surface.

Table 1. Mean hydrodynamic diameter and zeta potential of gold nanoparticles.

\begin{tabular}{|c|c|c|c|c|c|}
\hline Sample & AuNP conc. [nM] & BTZ conc. $[\mu \mathrm{M}]$ & Size $[\mathrm{nm}]$ & Pdl & Zeta potential [mV] \\
\hline AuNPs & 13.8 & 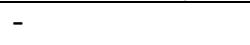 & $35 \pm 1.0$ & 0.6 & $-40 \pm 1.0$ \\
\hline PEGAuNPs * & 14.6 & - & 38 & 0.4 & -41 \\
\hline PEGAuNPs ** & 10.0 & - & $38 \pm 0.7$ & 0.6 & $-40 \pm 0.5$ \\
\hline PEGAuNP+BTZ & 13.6 & 150 & $39 \pm 0.4$ & 0.6 & $-18 \pm 0.4$ \\
\hline
\end{tabular}

$* \ln \mathrm{H}_{2} \mathrm{O} ; * * \ln 0.1 \mathrm{M}$ PBS 
In the UV-Vis spectra of PEGAuNPs and PEGAuNPs+BTZ a distinctive and characteristic surface plasmon resonance band centered at $526.5 \mathrm{~nm}$ was observed, with the appearance of new band at $270 \mathrm{~nm}$, characteristic for BTZ, in the latter (Figure 4). The results confirm the gold nanoparticle formation and BTZ inclusion into PEGAuNPs.

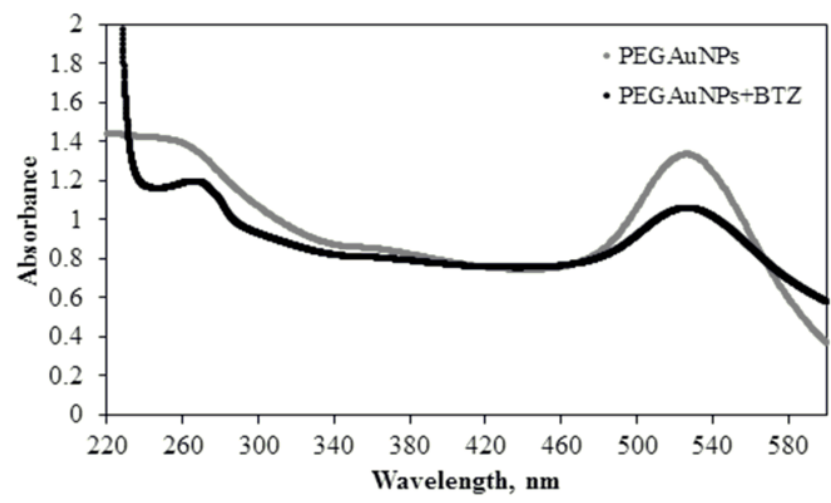

Figure 4. UV-Vis spectra of PEGAuNPs and PEGAuNPs+BTZ.

The FTIR spectra of BTZ, PEGAuNPs and PEGAuNPs+BTZ were measured and analyzed to further explore the inclusion and interaction of BTZ with PEGylated gold nanoparticles (Figure 5). The spectra of both PEGAUNPs and PEGAUNPs+BTZ have shown typical characteristic vibrations for the PEG functional groups at 1091 and $1117 \mathrm{~cm}^{-1}$ (C-O-C asymmetric, PEG chains), $1710 \mathrm{~cm}^{-1}$ (C=O, PEG layer), $1250 \mathrm{~cm}^{-1}$ (C-O, carbonyl), $1597 \mathrm{~cm}^{-1}$ (C=O asymmetric, AuNPs), $1392 \mathrm{~cm}^{-1}\left(\mathrm{O}-\mathrm{H}\right.$ in plane bend) and $3420 \mathrm{~cm}^{-1}(\mathrm{O}-\mathrm{H}, \mathrm{H}-$ bond). Characteristic bands at 603 and $697 \mathrm{~cm}^{-1}$ (B-C stretching), 900-700 cm-1 (=C-H aromatic, out-of plane bending), 1149 and $1198 \mathrm{~cm}^{-1}$ (B-O-H), $1274 \mathrm{~cm}^{-1}$ (C-N stretch), $1382 \mathrm{~cm}^{-1}$ (strong B-O stretching), 1464 and $1579 \mathrm{~cm}^{-1}$ (aromatics $\mathrm{C}=\mathrm{C}$ stretch bands), $1516 \mathrm{~cm}^{-1}$ (from N-H bending for secondary amide), 1652 (C=O stretching band), $2941 \mathrm{~cm}^{-1}(\mathrm{C}-\mathrm{H})$ and $3230 \mathrm{~cm}^{-1}$ (broad O-H stretching mode from boronic acid) were registered in the FTIR spectrum of BTZ (Figure 5(3)). In the IR spectrum of PEGAuNPs+BTZ (Figure 5(2)) characteristic bands for the broad $\mathrm{O}-\mathrm{H}$ stretching mode from boronic acid $\left(3230 \mathrm{~cm}^{-1}\right), \mathrm{C}-\mathrm{H}$ stretch of aromatic $\left(2941 \mathrm{~cm}^{-1}\right)$ and for the N-H bend of amide $\left(1549 \mathrm{~cm}^{-1}\right)$ groups of BTZ appear.

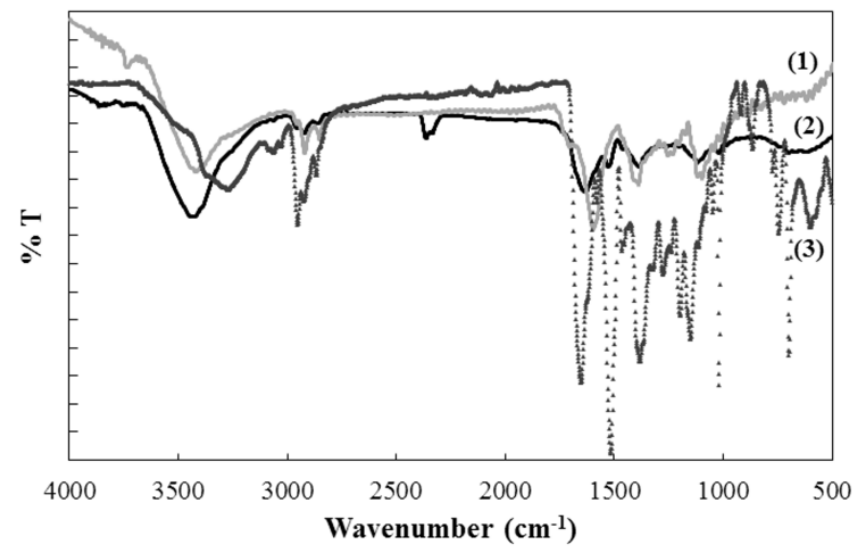

Figure 5. FTIR spectra of (1) PEGAuNPs, (2) PEGAuNPs+BTZ, (3) free BTZ. The spectra are scaled for better visualization.

A small shift of some absorption peaks of $\mathrm{BTZ}(\mathrm{O}-\mathrm{H}$ in plane bend and $-\mathrm{C}-\mathrm{O}-$ asymmetric, PEG chains) and change in band line shape were observed. The shift in the carbonyl stretch vibrations and $\mathrm{C}=\mathrm{O}$ stretching $\left(1628 \mathrm{~cm}^{-1}\right)$ in PEGAuNPs+BTZ, and the disappearance of the $1250 \mathrm{~cm}^{-1}$ resonance suggests an electrostatic attraction between the oppositely charged PEGAuNPs and BTZ species. This assumption is consistent with the significant change of the zeta potential of PEGAuNPs in the presence of BTZ.

Solution state ${ }^{1} \mathrm{H}$ NMR spectroscopy was used to study the composition and structure of BTZ loaded PEGAuNPs and to define the nature of drug-PEGAuNPs interactions, mechanisms of BTZ immobilization 
and location in the surface modified AuNPs. The study was focused on analysis of the induced alterations of a number of NMR parameters, such as chemical shifts, line shape, spin-lattice relaxation time $\left(T_{1}\right), N O E$ and translational diffusion of the protons of BTZ and PEG as a result of the drug loading of PEGAuNPs. Diffusion ordered spectroscopy (DOSY), measurements of the relaxation times and nuclear Overhauser effects were used to define possible BTZ-PEGAuNPs interactions and binding capability of PEGAuNPs. The NMR study was first carried out on BTZ, PEG and PEGAuNPs samples to fully characterize the structures and afterwards on PEGAuNPs+BTZ thus allowing the analysis of changes, induced by the drug inclusion into PEGAUNPs, on characteristic NMR parameters of the species present in the sample. The NMR data obtained for PEGAuNPs and PEGAuNPs+BTZ were compared to the hydrodynamic diameters and surface charges determined for the corresponding species.

Representative ${ }^{1} \mathrm{H}$ NMR spectra of BTZ, PEG, PEGAuNPs and PEGAuNPs+BTZ in $\mathrm{H}_{2} \mathrm{O}$ with $10 \% \mathrm{D}_{2} \mathrm{O}$ at $300 \mathrm{~K}$, with the assignment of the characteristic proton resonances in the molecules are presented in Figure 6.

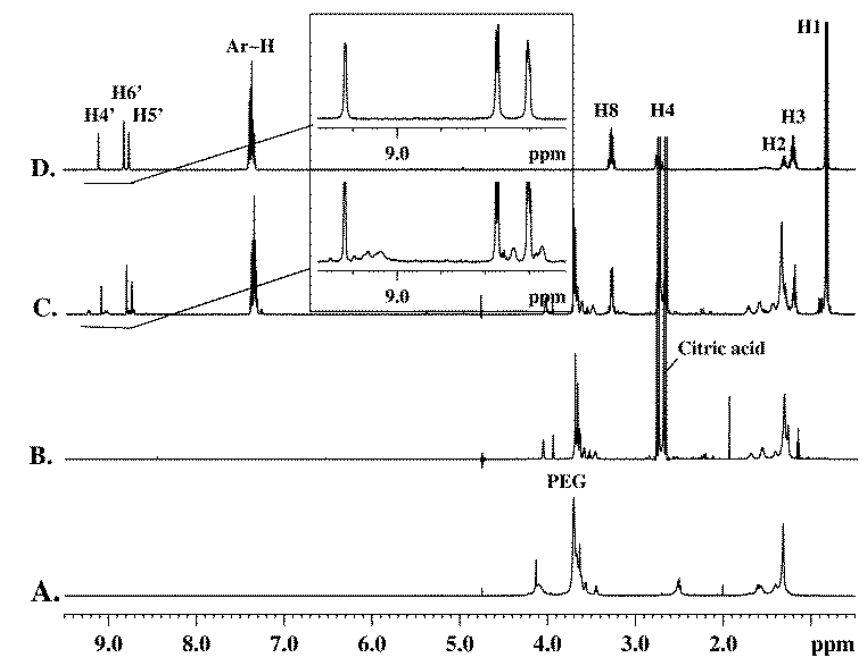

Figure 6. $600.13 \mathrm{MHz}{ }^{1} \mathrm{H}$ NMR spectra of: A) PEG; B) PEGAUNPs; C) PEGAuNPs+BTZ and D) BTZ. The assignment of BTZ signals is included.

The assignment of the resonance signals in the ${ }^{1} \mathrm{H}$ NMR spectra of BTZ and PEGAuNPs+BTZ was based on the analysis of the one- $\left(1 \mathrm{D} ;{ }^{1} \mathrm{H}\right)$ and two-dimensional $\left(2 \mathrm{D} ;{ }^{1} \mathrm{H} /{ }^{1} \mathrm{H}\right.$ COSY,$\left.{ }^{1} \mathrm{H} /{ }^{13} \mathrm{C} \mathrm{HSOC} \& \mathrm{HMBC}\right) \mathrm{NMR}$ spectroscopic data. Proton chemical shifts, of BTZ, in the free form and included into PEGAuNPs are shown in Table 2.

Table 2. ${ }^{1} \mathrm{H}$ NMR chemical shifts and spin-lattice relaxation times (T1) of the free BTZ and BTZ included in PEGAuNPs $\left(\mathrm{BTZ}^{*}\right.$ and $\left.\mathrm{BTZ}^{* *}\right)$.

\begin{tabular}{|c|c|c|c|c|c|c|}
\hline \multirow{2}{*}{${ }^{1} \mathrm{H}$} & \multicolumn{3}{|c|}{ Chemical shifts (ppm) } & \multicolumn{3}{|c|}{ Spin-lattice relaxation times, (T1; [s]) } \\
\hline & BTZ & BTZ* & BTZ** & BTZ & BTZ* $^{*}$ & BTZ** \\
\hline H1a & 0.820 & 0.820 & - & 0.620 & 0.559 & \\
\hline $\mathrm{H} 1 \mathrm{~b}$ & 0.832 & 0.834 & - & 0.620 & 0.559 & - \\
\hline $\mathrm{H} 2$ & 1.307 & 1.321 & - & 0.445 & 0.379 & - \\
\hline H3 & 1.205 & 1.218 & - & 0.416 & 0.364 & - \\
\hline H4 & 2.752 & 2.760 & - & 1.010 & 0.700 & 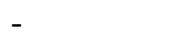 \\
\hline H7 & 4.963 & 4.949 & - & 1.205 & - & - \\
\hline $\mathrm{H} 8 \mathrm{a}$ & 3.249 & 3.231 & 3.119 & 0.386 & 0.297 & - \\
\hline $\mathrm{H} 8 \mathrm{~b}$ & 3.292 & 3.284 & 3.119 & 0.365 & 0.297 & 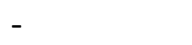 \\
\hline $\mathrm{Ar}$ & 7.348 & 7.348 & 7.258 & 1.443 & & - \\
\hline$H 4^{\prime}$ & 9.099 & 9.095 & 9.057 & 6.032 & 4.800 & - \\
\hline $\mathrm{H}^{\prime}$ & 8.752 & 8.752 & 8.729 & 3.603 & 2.859 & 2.920 \\
\hline$H 6^{\prime}$ & 8.810 & 8.809 & 8.781 & 3.341 & 2.910 & 2.919 \\
\hline
\end{tabular}

In the ${ }^{1} \mathrm{H}$ NMR spectrum of PEGAuNPs+BTZ two sets of resonance signals were observed, belonging to BTZ, both shifted with respect to the corresponding signals of the free drug. The results confirmed the existence 
of two forms of BTZ, but no evidence was found for BTZ chemically bonded to the PEGAuNPs. The alterations in the chemical shift, line shape and intensity of the resonance signals belonging to the two forms of BTZ indicate the existence of at least two different microenvironments in PEGAuNPs accessible for the drug molecules. Resonance signals, slightly broadened and shifted $(<0.02 \mathrm{ppm})$ relative to the corresponding signals of the free drug were registered for one of the BTZ forms, further marked as BTZ*. These chemical shift and spectral line shape alterations indicate the existence of intermolecular interactions of drug molecules most probably with the polymer layer of the PEGAuNPs. The second set of weak, much broader and upfield shifted BTZ signals in the ${ }^{1} \mathrm{H}$ NMR spectrum of PEGAuNPs+BTZ, were attributed to protons of drug molecules located in close proximity to the metal core of the PEGAuNPs. It was suggested that the negatively charged AuNP surface attracts the positively charged drug molecules generated at low $\mathrm{pH}(\mathrm{pH} \mathrm{5.3)}$ in the solution, facilitating their adsorption on the nanoparticles. The NMR signals of these species adsorbed on the surface of the nanoparticles are drastically broadened due to reduced mobility of drug molecules and large correlation time. The observed significant line broadening and upfield chemical shift of the second set of BTZ resonance signals are consistent with drug molecules being adsorbed on AuNP surface as a result of electrical charge or dipole/dipole interactions. This second drug form is denoted as $\mathrm{BTZ}^{* *}$. The mole fractions of the two forms of $\mathrm{BTZ}, \mathrm{BTZ}^{*}$ and $\mathrm{BTZ}^{* *}$, observed in the ${ }^{1} \mathrm{H}$ NMR spectra of PEGAuNPs+BTZ were estimated by the integral intensity of the resonance signals from the aromatic spectral area $\left(\mathrm{H}^{\prime}-\mathrm{H} 6^{\prime}\right)$. It was found that the fraction of $\mathrm{BTZ}{ }^{*}$ dominates and represents around $80 \%$ from the total amount of the drug in the sample.

For the studied DDS, NMR relaxation measurements can provide structural and dynamic information about molecules and molecular conjugations. ${ }^{49}$ The rate of relaxation is typically influenced by the physical properties of the molecule and the sample, so a study of relaxation phenomena can lead to information on these properties and can be used to probe the internal motions of the species in DDS. The relaxation of the nuclear magnetization to its equilibrium is governed by the spin-lattice $\left(T_{1}\right)$ and spin-spin $\left(T_{2}\right)$ relaxations and is related via correlation times to the mobility of the molecules and may be used to estimate the possibility for intermolecular aggregate formation. The spin-spin relaxation time typically decreases as the correlation time increases, resulting in NMR spectral line broadening. However, $T_{1}$ relaxation is optimal if the average rate of molecular reorientation in space matches the Larmour frequency of the spectrometer. Typically, for larger molecules the molecular motions become slower (longer correlation times), resulting in more effective (shorter) spin-lattice relaxation times. ${ }^{49}$, 50 Therefore, measurements of the spin-lattice relaxation times may be used to explore the existence of molecular interactions in the studied DDS. ${ }^{34,39}$

Considering that the relaxation times of the nuclei are affected by the physical properties of molecules and/or existence of molecular interactions, the spin-lattice relaxation times of protons belonging to BTZ in its free form and incorporated in PEGAuNPs were measured and analyzed. The $T_{1}$ relaxation times of protons from the individual functional groups of BTZ, in the both samples vary according to their internal mobility. Nevertheless, an overall decreasing trend was observed in the spin-lattice relaxation times of BTZ protons in the PEGAuNPs+BTZ sample (Table 2). The increased rate of relaxation of BTZ protons was attributed to more effective dipole-dipole interactions between drug molecules and PEG coated AuNPs. The line broadening of BTZ resonances in ${ }^{1} \mathrm{H}$ NMR spectra of PEGAuNPs+BTZ and significantly increasing relaxation rates strongly suggest the successful inclusion of the drug molecules into PEGAuNPs and the existence of BTZ-NP interactions and chemical exchange processes in which the protons exchange between two or more environments.

Diffusion ordered ( ${ }^{1} \mathrm{H}$ DOSY) and Saturation Transfer Difference (STD) NMR spectroscopy were applied to further explore the interactions of BTZ with PEGAUNPs, the mode of drug incorporation and location within the nanoparticles. DOSY is particularly sensitive technique to structural and dynamical changes at the molecular level; it can provide useful information about the structural composition of nano-systems and allows the amount of drug molecules effectively associated with the NPs to be quantified. ${ }^{34,51}$ STD-NMR technique based on the intermolecular NOE, has emerged as one of the most widespread NMR methods for studying ligand-receptor interactions and can be useful tool for detection of interactions between small drugs and nanoparticles and to identify the localization of diverse drugs in nanosystems. ${ }^{34,52,53}$

From ${ }^{1} \mathrm{H}$ DOSY experiments, the diffusion coefficients (D) of BTZ and PEG in PEGAuNPs and PEGAuNPs+BTZ were measured and compared with the corresponding values obtained for the free BTZ and PEG. The D values of BTZ and PEG were determined from the resonance signals of $\mathrm{H}^{\prime}{ }^{\prime}-\mathrm{H} 6^{\prime}$ for $\mathrm{BTZ}$ and $-\mathrm{OCH}_{2}$ protons of PEG in ${ }^{1} \mathrm{H}$ NMR spectra. The results are presented in Figure 7. 


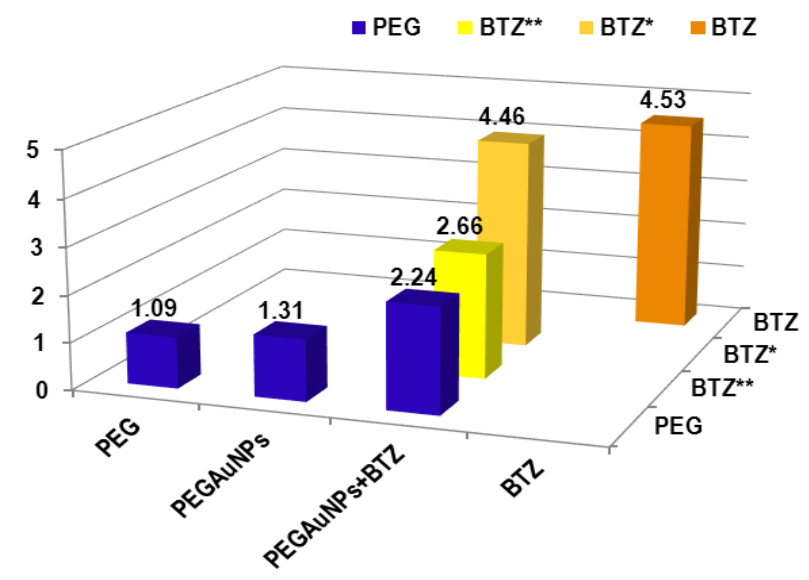

Figure 7. Diffusion coefficients $\left(D \times 10^{10} \mathrm{~m}^{2} \mathrm{~s}^{-1}\right)$ of $B T Z$ and PEG in the samples studied. All values are corrected to $\mathrm{D}_{\text {TSP }} ; \mathrm{BTZ}$ - stands for the free Bortezomib.

The diffusion coefficients measured for the two forms of BTZ (BTZ* and BTZ**) existing in the drug loaded PEGAuNPs were found to be significantly different as can be seen from Figure 7 . The diffusion coefficient of BTZ* was found to be similar to the corresponding value of the free drug therefore the presence of only weak interactions with PEGAuNPs were assumed. In contrast, the diffusion coefficient measured for the second drug form (BTZ**) present in the sample was found to be significantly lower when compared to the value of $\mathrm{D}$ of the free drug and $\mathrm{BTZ}^{*}$, but similar to the corresponding value registered for PEG. The significant reduction of $\mathrm{BTZ}^{* *}$ diffusion to a value characteristic for the functionalized NPS is a strong indication of an association process between these species. The diffusion behaviour of BTZ* and BTZ** could be interpreted as a fast exchange between two sites, i.e. bound to the NPs and free or physically entrapped drug molecules ${ }^{38,51}$. In this case, the observed diffusion coefficients are weighted-average values between the free and bound drug species and if we consider fast exchange between these two states, the mole fractions of the free and bound drug molecules could be calculated using the following equation:

$D=f_{\text {free }} D_{\text {free }}+f_{\text {bound }} D_{\text {bound }}$

where $f$ and $D$ denote the molecular fractions and diffusion coefficients of the free and bound drug molecule and $f_{\text {free }}+f_{\text {bound }}=1$. The fractions of the bound BTZ* and BTZ** in the BTZ loaded PEGAuNPs were estimated using the diffusion coefficient of the free BTZ ( $\left.D_{\text {free }}\right)$ measured separately and assuming the diffusion coefficient of the bound drug molecules ( $D_{\text {bound }}$ ) to be equal to that of the PEG in the sample. The fraction of the bound drug molecules for the two forms BTZ* and BTZ** in the PEGAuNPs+BTZ was found to be $3 \%$ and $82 \%$, respectively.

It is proposed that the BTZ* molecules in PEGAUNPs+BTZ are distributed in and weakly interact with the polymer chains from the PEG layer coating of the AuNPs, whereas BTZ** species are predominantly adsorbed on the surface of AuNPs. These assumed locations of BTZ* and BTZ** with respect to the PEGAuNPs structure could explain the decrease of the negative surface charge of PEGAuNPs+BTZ, the significant alterations in characteristic NMR parameters of BTZ** and the weak changes of those for BTZ*. The STD-NMR technique was further used to verify the assumption that the BTZ* form corresponds to molecules distributed in the polymer layer of PEGAuNPs. Bearing in mind the results from the aforementioned experiments and the ability of the STD technique to detect weak ligand-receptor bindings, experiments were conducted to explore possible interactions of drug molecules with nanoparticles, and more specifically with PEG chains attached to the NP surface. It was expected that BTZ protons that are in close contact with the polymer chains of PEGAuNPs will receive saturation transfer from the polymer via spin diffusion, through the NOE when selectively saturated. Protons that are either less or not involved in a binding process reveal no STD-NMR signals. The STD experiment was acquired by subtracting a spectrum recorded with selective saturation of the protons belonging to the PEG chains (at $3.7 \mathrm{ppm},-\mathrm{OCH}_{2}$ protons) from a spectrum recorded without saturation (off-resonance spectrum) of the polymer. The STD-NMR and reference ${ }^{1} \mathrm{H}$ NMR spectra of BTZ loaded PEGAuNPs are presented in Figure 8. The results confirm weak 
intermolecular interactions between the aromatic, methyl and methylene ( $\mathrm{H} 8)$ protons of $\mathrm{BTZ}^{*}$ and

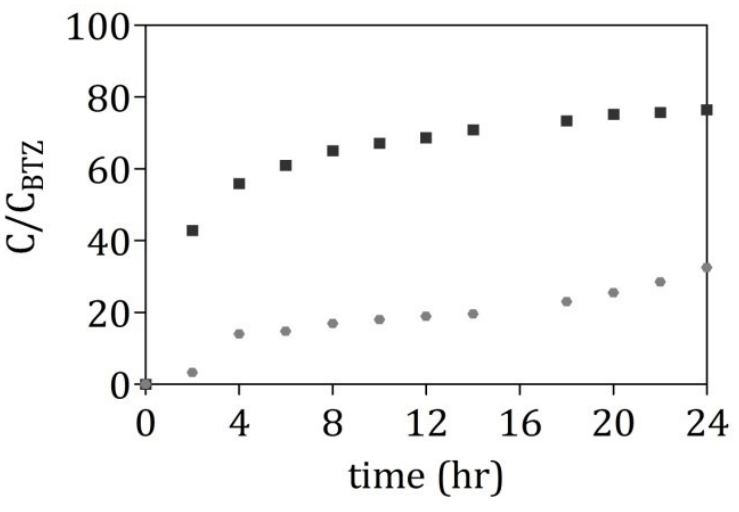
polymer chains of PEG (Figure 8).

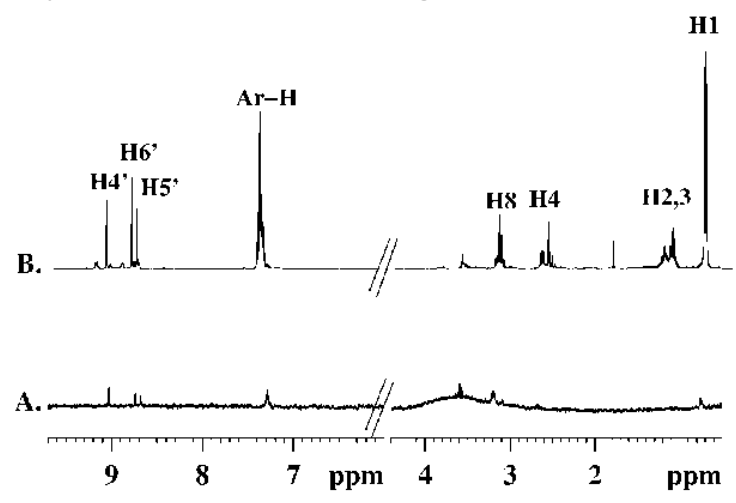

Figure 8. $400.15 \mathrm{MHz}{ }^{1} \mathrm{H}$ STD-NMR experiment of $\mathrm{BTZ}$ loaded PEGAuNPs in $\mathrm{H}_{2} \mathrm{O}$ (with $10 \% \mathrm{D}_{2} \mathrm{O}$ ): A) STD-NMR spectrum; B) Reference spectrum.

Figure 9. Release profiles of BTZ ( $\square)$ and BTZ+PEGAuNPs ( $)^{\circ}$ in PBS $0.01 \mathrm{M}$. $\mathrm{C}_{\max }$ corresponds to the total amount of BTZ added.

The in vitro release profile of BTZ was studied by dialysis, at pH 7.4 in PBS $0.01 \mathrm{M}$, and the results suggested significant changes on the diffusion of free BTZ and BTZ loaded PEGAuNPs (Figure 9). ${ }^{41}$ BTZ loaded PEGAuNPs exhibits, in the first two hours, a delay on the drug released. The amount of BTZ released based on total mixed BTZ was $15 \%$ after 4 hours in comparison with $56 \%$ of free BTZ. These results are compatible with the diffusion behaviour of the two drug forms (BTZ* and BTZ**) defined in the BTZ loaded PEGAuNPs.

The registered drug release profile of BTZ loaded PEGAUNPs reveals the potential of this nanoparticulate drug delivery system for sustained delivery/release of BTZ in vitro.

\section{Conclusions}

This work presents a new approach based on NMR spectroscopy and a number of complementary analytical techniques such as FTIR, UV-Vis, DLS and TEM for detailed structural and physicochemical characterization of surface modified gold nanoparticles, designed for drug delivery of the proteasome inhibitor Bortezomib (BTZ) in cancer therapy. The results confirm the formation of PEG surface functionalized gold nanoparticles with a spherical shape, average size around $40 \mathrm{~nm}$ and negative surface charge, which incorporate the proteasome inhibitor BTZ.

Moreover, the NMR results reveal the existence of two different BTZ microenvironments in the PEG coated AuNPs and different mechanisms of drug inclusion. The analysis of ${ }^{1} \mathrm{H}$ NMR spectra confirmed the coexistence of two BTZ forms, BTZ* and BTZ**, in the PEGAuNPs characterized by different chemical shifts, line shape and quantitative distribution. The chemical shift and line broadening of both $\mathrm{BTZ}^{*}$ and $\mathrm{BTZ} * *$ resonances in ${ }^{1} \mathrm{H}$ NMR spectra of PEGAuNPs+BTZ and significantly increasing relaxation rates show not only successful inclusion of the drug molecules into PEGAuNPs, but also the existence of BTZ - PEGAuNP interactions and chemical exchange processes.

Diffusion ordered NMR spectroscopy combined with studies of the nuclear Overhauser effect have provided valuable information on BTZ-PEGAuNPs interactions and the mechanism of drug incorporation and location inside the PEGAuNPs. The DOSY and STD-NMR results indicate that the BTZ* molecules in PEGAuNPs+BTZ are distributed in and weakly interact with the polymer chains from the PEG layer coating of the AuNPs, whereas BTZ** species are predominantly adsorbed on the surface of AuNPs. The defined locations of $\mathrm{BTZ}^{*}$ and $\mathrm{BTZ}^{* *}$ with respect to the PEGAuNPs structure are consistent with the decrease of the negative surface charge of BTZ loaded PEGAuNPs, the significant alterations in characteristic NMR parameters of BTZ** and the weak changes of those for BTZ*.

The drug locations and interactions define the overall structure of DDS and affect their capacity for sustained drug release, targeted drug delivery, and hence efficacy.

The present study demonstrates the utility of NMR spectroscopy for detailed structural characterization of nanomaterials for biomedical applications. Moreover, it is demonstrated that the combined application of 
NMR spectroscopy with FTIR, UV-Vis, DLS and TEM can provide valuable information regarding important physicochemical properties of DDS, which affect their therapeutic efficacy. The NMR approach described in this work can be applied to a wide variety of nano-scale drug delivery systems.

\section{Acknowledgments}

This work received financial support from the European Union (FEDER funds through COMPETE) and National Funds (FCT, Fundação para a Ciência e Tecnologia) through projects Pest-C/EQB/LA0006/2013. The NMR spectrometers were purchased under the framework of QREN, through project NORTE-07-0162FEDER-000048, and are part of the Portuguese National NMR Network. To all financing sources the authors are greatly indebted. We gratefully acknowledge funding received from TRANSCAN-FCT (research project TRANSCAN/0001/2012) and Portuguese Cancer League.

\section{References}

1. C. S. S. R. Kumar, Nanomaterials for Cancer Therapy, WILEY-VCH Verlag GmbH \& Co. KGaA, 2006.

2. $\quad$ A. Z. Wang, R. Langer and O. C. Farokhzad, Annu Rev Med, 2012, 63, 185-198.

3. J.-W. Yoo, N. Doshi and S. Mitragotri, Advanced Drug Delivery Reviews, 2011, 63, 1247-1256.

4. A. Prokop and J. M. Davidson, Journal of pharmaceutical sciences, 2008, 97, 3518-3590.

5. $\quad$, ed. Y. Yeo, John Wiley \& Sons, 2013, p. 324.

6. M. M. d. Villiers, P. Aramwit and G. S. Kwon, NANOTECHNOLOGY IN DRUG DELIVERY 2009.

7. J. A. Kim, C. Aberg, A. Salvati and K. A. Dawson, Nat Nano, 2012, 7, 62-68.

8. G. T. Cai Weibo, Hong Hao, Sun Jiangtao, Nanotechnology, Science and Applications, 2008, 1, 17-32.

9. J. Li, S. Gupta and C. Li, Quantitative Imaging in Medicine and Surgery, 2013, 3, 284-291.

10. E. Boisselier and D. Astruc, Chemical Society Reviews, 2009, 38, 1759-1782.

11. J. Lee, D. K. Chatterjee, M. H. Lee and S. Krishnan, Cancer Letters, 2014, 347, 46-53.

12. L. Vigderman and E. R. Zubarev, Advanced Drug Delivery Reviews, 2013, 65, 663-676.

13. A. de la Zerda, S. Prabhulkar, V. L. Perez, M. Ruggeri, A. S. Paranjape, F. Habte, S. S. Gambhir and R. M. Awdeh, Clinical \& Experimental Ophthalmology, 2015, DOI: 10.1111/ceo.12299, n/a-n/a.

14. X. Huang and M. A. El-Sayed, Journal of Advanced Research, 2010, 1, 13-28.

15. T. Curry, R. Kopelman, M. Shilo and R. Popovtzer, Contrast Media \& Molecular Imaging, 2014, 9, 53-61.

16. W.-S. Kuo, Y.-T. Chang, K.-C. Cho, K.-C. Chiu, C.-H. Lien, C.-S. Yeh and S.-J. Chen, Biomaterials, 2012, 33, 3270-3278.

17. Y. Q. Du, X. X. Yang, W. L. Li, J. Wang and C. Z. Huang, RSC Advances, 2014, 4, 34830-34835.

18. F. Wang, Y.-C. Wang, S. Dou, M.-H. Xiong, T.-M. Sun and J. Wang, ACS Nano, 2011, 5, 3679-3692.

19. C. Tomuleasa, O. Soritau, A. Orza, M. Dudea, B. Petrushev, O. Mosteanu, S. Susman, A. Florea, E. Pall, M. Aldea, G. Kacso, V. Cristea, I. Berindan-Neagoe and A. Irimie, J Gastrointestin Liver Dis, 2012, 21, 187196.

20. P. K. Jain, I. H. El-Sayed and M. A. El-Sayed, Nano Today, 2007, 2, 18-29.

21. B. D. Chithrani and W. C. W. Chan, Nano Letters, 2007, 7, 1542-1550.

22. B. Kim, G. Han, B. J. Toley, C.-k. Kim, V. M. Rotello and N. S. Forbes, Nat Nano, 2010, 5, 465-472.

23. H. Ali-Boucetta and K. Kostarelos, Adv Drug Deliv Rev, 2013, 65, 2111-2119.

24. R. R. Arvizo, S. Rana, O. R. Miranda, R. Bhattacharya, V. M. Rotello and P. Mukherjee, Nanomedicine, 2011, 7, 580-587.

25. K. Kalishwaralal, S. Sheikpranbabu, S. BarathManiKanth, R. Haribalaganesh, S. Ramkumarpandian and S. Gurunathan, Angiogenesis, 2011, 14, 29-45.

26. R. R. Arvizo, S. Rana, O. R. Miranda, R. Bhattacharya, V. M. Rotello and P. Mukherjee, Nanomedicine: Nanotechnology, Biology and Medicine, 2011, 7, 580-587.

27. L. Brannon-Peppas and J. O. Blanchette, Adv Drug Deliv Rev, 2004, 56, 1649-1659.

28. P. Mukherjee, R. Bhattacharya, P. Wang, L. Wang, S. Basu, J. A. Nagy, A. Atala, D. Mukhopadhyay and S. Soker, Clinical Cancer Research, 2005, 11, 3530-3534.

29. S. Jelveh and D. B. Chithrani, Cancers, 2011, DOI: 10.3390/cancers3011081.

30. M. R. Papasani, G. Wang and R. A. Hill, Nanomedicine: Nanotechnology, Biology and Medicine, 2012, 8, 804-814. 
31. A. Kumar, X. Zhang and X. J. Liang, Biotechnol Adv, 2013, 31, 593-606.

32. $\quad$ ed. D. T. Yashwant Pathak, CRC Press, 2009, p. 416.

33. Z. Hens and J. C. Martins, Chemistry of Materials, 2013, 25, 1211-1221.

34. R. López-Cebral, M. Martín-Pastor, B. Seijo and A. Sanchez, Progress in Nuclear Magnetic Resonance Spectroscopy, 2014, 79, 1-13.

35. L. E. Marbella and J. E. Millstone, Chemistry of Materials, 2015, DOI: 10.1021/cm504809c.

36. T. Brand, E. J. Cabrita and S. Berger, Progress in Nuclear Magnetic Resonance Spectroscopy, 2005, 46, 159-196.

37. Y. Cohen, L. Avram and L. Frish, Angewandte Chemie International Edition, 2005, 44, 520-554.

38. K. I. Momot and P. W. Kuchel, Concepts in Magnetic Resonance Part A, 2003, 19A, 51-64.

39. G. Ivanova, M. Simeonova, E. J. Cabrita and M. Rangel, The Journal of Physical Chemistry B, 2011, 115, 902-909.

40. M. Simeonova, M. Rangel and G. Ivanova, Physical Chemistry Chemical Physics, 2013, 15, 16657-16664.

41. S. C. Coelho, S. Rocha, P. Juzenas, P. Sampaio, G. M. Almeida, F. S. Silva, M. C. Pereira and M. A. N. Coelho, Expert Opinion on Drug Delivery, 2013, 10, 1345-1352.

42. S. C. Coelho, S. Rocha, M. C. Pereira, P. Juzenas and M. A. Coelho, Journal of biomedical nanotechnology, 2014, 10, 717-723.

43. S. E. Canfield, K. Zhu, S. A. Williams and D. J. McConkey, Mol Cancer Ther, 2006, 5, 2043-2050.

44. J. Turkevich, P. C. Stevenson and J. Hillier, Discussions of the Faraday Society, 1951, 11, 55-75.

45. T. L. Hwang and A. J. Shaka, Journal of Magnetic Resonance, Series A, 1995, 112, 275-279.

46. M. Mayer and B. Meyer, Angewandte Chemie International Edition, 1999, 38, 1784-1788.

47. M. Mayer and B. Meyer, Angewandte Chemie, 1999, 111, 1902-1906.

48. D. H. Wu, A. D. Chen and C. S. Johnson, Journal of Magnetic Resonance, Series A, 1995, 115, 260-264.

49. V. I. Bakhmutov, Practical NMR Relaxation for Chemists, John Wiley \& Sons, Ltd, U.K., 2005.

50. J. Keeler, Understanding NMR Spectroscopy, JohnWiley \& Sons: Chichester, U.K., 2nd Edition edn., 2010.

51. Y. Cohen, L. Avram and L. Frish, Angew Chem Int Ed Engl, 2005, 44, 520-554.

52. B. Meyer and T. Peters, Angew Chem Int Ed Engl, 2003, 42, 864-890.

53. A. Viegas, J. Manso, F. L. Nobrega and E. J. Cabrita, Journal of Chemical Education, 2011, 88, 990-994. 